\title{
GENERAL TRINOMIALS HAVING SYMMETRIC GALOIS GROUP
}

\author{
JOHN HOWARD SMITH
}

\begin{abstract}
Let $a$ and $b$ be independent transcendentals over a field $F$ and let $n>k$ be integers $>1$ with $n, k, n-k$ and the characteristic of $F$ pairwise relatively prime. Then the Galois group of $x^{n}+a x^{k}+b$ over $F(a, b)$ is $S_{n}$.
\end{abstract}

It is well known (e.g. p. 175 of [3]) that the Galois group of the general polynomial, $X^{n}+a_{1} X^{n-1}+\cdots+a_{n}$ is the full symmetric group on the roots. Uchida [2] has shown that with suitable conditions on the characteristic the same is true of the polynomial $X^{n}+a X+b$. In this note we extend Uchida's methods and result to the case $X^{n}+a X^{k}+b$, where $(k, n)=1$ (obviously a necessary condition).

LEMMA 1. Let $a$ and $b$ be independent variables over a field $F$. Let $(m, n)=$ $1, m, n \in Z^{+}$and let $r m+s n=1, r, s \in Z$. Let $c=b^{s} a^{r}$ and let the ring $R=F[a, b, c]=F[a, b] \subset F(a, b)$. Then for any nonzero $u$, $v$ in $F$, if $P$ is the principal ideal $\left(u a^{n}-v b^{m}\right)$ in $R$, then $R / P$ is the polynomial ring over $F$ in one variable. (In particular $P$ is prime.)

Proof. If $\bar{a}, \bar{b}, \bar{c}$ denote the images of $a, b, c$ modulo $P$ then $\bar{b}=$ $(u / v)^{r} \bar{c}^{n}, \bar{a}=(v / u)^{s} \bar{c}^{m}$ so $R / P=F[\bar{c}]$. To see that $\bar{c}$ satisfies no polynomial over $F$ we note that since $R$ has transcendence degree 2 over $F, R / P$ must have transcendence degree (at least) 1.

Lemma 2. Let $a, b$ be independent transcendentals over a field $F$ and let $K=F(a, b)$. Then $x^{n}+a X^{k}+b$ is irreducible in $K[X]$.

Proof. It suffices to show irreducibility for some specialization of the coefficients; in this case let $a=0$ and apply the Eisenstein criterion.

Lemma 3. Let $B=\left(b_{i j}\right)$ be an $n \times n$ matrix such that $b_{i j}=0$ unless $j-i \equiv$ $m$ or $k(\bmod n)$. Suppose further that $(m-k, n)=1$. Then the only nonzero terms in the determinant are $\Pi_{i} b_{i i+m}$ and $\Pi_{i} b_{i i+k}$ (with appropriate signs).

Proof. Elementary.

Received by the editors December 10, 1974 and, in revised form, June 13, 1975 and February 24, 1976.

AMS (MOS) subject classifications (1970). Primary 12A20, 12A55, $12 \mathrm{~F} 10$. 
LEMMA 4. If $(k, n)=1$ and the polynomial $X^{n}+a X^{k}+b$ with coefficients in a field $K$ is irreducible, then its discriminant is:

$$
(-1)^{n(n-1) / 2} b^{k-1}\left[n^{n} b^{n-k}+(-1)^{(n+1) k}(n-k)^{n-k} k^{k} a^{n}\right] .
$$

(See Note added in proof.)

SKETCH OF PROOF. If the roots of $f(X)=0$ are $\alpha=\alpha_{1}, \alpha_{2}, \ldots, \alpha_{n}$ the discriminant is

$$
(-1)^{n(n-1) / 2} \Pi_{i} f^{\prime}\left(\alpha_{i}\right)
$$

Multiplication by $f^{\prime}(\alpha)$ is a $K$-linear map of $K[\alpha]$ to itself whose determinant is the norm of $f^{\prime}(\alpha)$ or $\prod_{i} f^{\prime}\left(\alpha_{i}\right)$.

The determinant is computed with respect to the basis $1, \alpha, \ldots, \alpha^{n-1}$ using Laplace expansion on the first column, elementary row operations, and Lemma 3.

Lemma 5. Suppose that the characteristic of $F$ is prime to $n, k$ and $n-k$ and that $(k, n)=1$. Then, if $a, b$ are independent over $F$, the Galois group of $f(X)=X^{n}+a X^{k}+b$ over $K=F(a, b)$ contains a transposition.

Proof. Since $n f(X)-X f^{\prime}(X)=(n-k) a X^{k}+n b=h(X)$, any common zeros of $f$ and $f^{\prime}$ are also zeros of $h$, i.e. $k$ th roots of $-n b /(n-k) a$.

We consider the ring $R=F[a, b, c]$ with $c$ as in Lemma 1 and the ideal (prime by Lemma 1) $P=\left(n^{n} b^{m}+(-1)^{(n+1) k} m^{m} k^{k} a^{n}\right)$, where $m=n-k$. Let $\bar{R}=R / P$ and let $\bar{K}$ be the field of quotients. The polynomial $\bar{f}(X)=X^{n}$ $+\bar{a} X^{k}+\bar{b}$ certainly has a repeated zero, $\bar{\alpha}$, in some extension field of $\bar{K}$ since its discriminant vanishes by Lemma 4 . On the other hand, if $\bar{\alpha}$ and $\bar{\beta}$ are repeated zeros of $\bar{f}$ then $\bar{\alpha}^{k}=\bar{\beta}^{k}=-n \bar{b} /(n-k) \bar{a}$ and $\bar{\alpha}^{n}=\bar{\beta}^{n}=$ $-k \bar{b} /(n-k)$, hence, since $(n, k)=1, \bar{\alpha}=\bar{\beta}$. Further a triple zero of $\bar{f}$ would be a double zero of $\bar{h}$, but the latter has distinct roots by our assumption on the characteristic.

Now an irreducible factor of $f$ cannot have a double root since the hypotheses imply that the characteristic is not 2 . If two irreducible factors have a root in common they are both linear.

Hence $\bar{f}=\bar{f}_{1} \ldots \bar{f}_{m}$ where the $f_{i}$ are monic and pairwise relatively prime in $\bar{K}[X], \bar{f}_{1}(X)=(X-\bar{\alpha})^{2}$ and $\bar{f}_{2}, \ldots, \bar{f}_{m}$ are irreducible. Since $\bar{R}$ is integrally closed in $\bar{K}$ by Lemma 1 the factorization can be carried out over $\bar{R}$.

By Hensel's Lemma we may then factor over the completion $K_{P}$ of $K$ with respect to the prime ideal $P$ into $f=f_{1_{-}} \ldots f_{m}$ with each $f_{i}$ having coefficients in the completion $R_{P}$ and reducing to $f_{i}$ modulo $P$.

Since the $\bar{f}_{i}$ are irreducible for $i \geqslant 2$ and have the same degrees as the $f_{i}$, the extensions generated over $K_{P}$ by roots of the latter are unramified. However since $P=\left(\Pi_{i<j}\left(\alpha_{i}-\alpha_{j}\right)\right)^{2}, P$ ramifies if we adjoin all the roots of $f$, it must 
ramify if we adjoin a root of $f_{1}$; in particular the latter is irreducible over $K_{P}$. Hence the inertia group of the splitting field of $f$ over $K_{P}$ leaves the roots of $f_{i}$ fixed for $i \geqslant 2$ and interchanges those of $f_{1}$. This completes the proof.

LemMA 6. Let $G$ be a transitive permutation group on $A=\left\{\alpha_{1}, \ldots, \alpha_{n}\right\}$. Suppose $G$ includes $\sigma$, which permutes $\left\{\alpha_{1}, \ldots, \alpha_{k}\right\}=A^{\prime}$ cyclicly, and $\tau$, which permutes $\left\{\alpha_{k+1}, \ldots, \alpha_{n}\right\}=A^{\prime \prime}$ cyclicly. (This says nothing about how $\sigma$ acts on $A^{\prime \prime}$ or $\tau$ on $A^{\prime}$.) Then if $(k, n)=1, G$ is primitive.

Proof. Suppose there is a system of imprimitivity. For each block $B$ let $B^{\prime}=A^{\prime} \cap B, B^{\prime \prime}=A^{\prime \prime} \cap B$. Neither is empty, for if, for example, $B^{\prime \prime}=\varphi$, i.e. $B \subset A^{\prime}$ then the blocks contained in $A^{\prime}$ would be a system of imprimitivity for the action of $H$, the subgroup generated by $\sigma$, on $A^{\prime}$. Hence the order of $B$, a divisor of $n$, would also divide $k$.

Now $H$ permutes the $B^{\prime}$ cyclicly, hence permutes the $B$ cyclicly. Hence, the number of blocks (a divisor of $n$ ), divides $k$. This is impossible since there is more than one block.

To apply Lemma 6 to our equation we look for $\sigma, \tau$ in the decomposition groups of appropriate valuations. For the sake of symmetry it will be convenient to work temporarily with $\alpha X^{n}+\beta X^{k}+\gamma$, which clearly has the same Galois group as the original. We first prove a form of Hensel's Lemma.

LEMMA 7. Let $F$ be a field, let $\alpha, \beta, \gamma$ be independent transcendentals over $F$ and let $A=F(\beta)[[\alpha, \gamma]]$ (power series in $\alpha, \gamma$ with coefficients in $F(\beta)$ ). Let $B=A /(\alpha \gamma)$ and for any $g(X) \in A[X]$ let $\bar{g}(X) \in B[X]$ denote the reduction. Suppose $k<n-k$. Then $f(X)=\alpha X^{n}+\beta X^{k}+\gamma$ can be factored $f(X)=$ $f_{1}(X) f_{2}(X)$, with $f_{1}(X), f_{2}(X) \in A[X], \bar{f}_{1}(X)=X^{k}+\overline{\gamma / \beta}, \bar{f}_{2}(X)=\bar{\alpha} X^{n-k}+$ $\bar{\beta}$.

Proof. We apply Bourbaki's form of Hensel's Lemma (p.215 of [1]). $A$ is a complete linearly topologized ring under the topology given by total order in $\alpha$ and $\gamma, M=(\alpha \gamma)$ is an ideal whose elements are topologically nilpotent. Further, $\bar{f}(X)=\bar{f}_{1}(X) \bar{f}_{2}(X)$ where $\bar{f}_{1}(X)=X^{k}+\bar{\gamma} / \bar{\beta}, \bar{f}_{2}(X)=\bar{\alpha} X^{n-k}+\bar{\beta}$ and these are strongly relatively prime since the ideal they generate in $B[X]$ contains $\overline{\alpha \beta} X^{k}+\overline{\alpha \gamma}=\overline{\alpha \beta} X^{k}$, hence $\bar{\alpha} X^{k}$, hence $\bar{\alpha} X^{n-k}$, hence $\bar{\beta}$, a unit. The existence of the desired $f_{1}(X), f_{2}(X)$ follows.

LeMma 8. Let $F, \alpha, \beta, \gamma, f$ be as in Lemma 7. Suppose the characteristic of $F$ is prime to $k$ and $n-k$ and that $n \neq 2 k$. Then the roots of $f$ may be so numbered that there are $\sigma, \tau$ in the Galois group of $f$ over $F(\alpha, \beta, \gamma)$ permuting $\alpha_{1}, \ldots, \alpha_{k}$ and $\alpha_{k+1}, \ldots, \alpha_{n}$, respectively, cyclicly.

Proof. Assume $k<n-k$. (The case $k>n-k$ is similar.) Let $A$ be as in Lemma 7, $L=F(\beta)\{\{\alpha, \gamma\}\}$ its field of quotients (formal Laurent series in $\alpha$ 
and $\gamma$ over $F(\beta)), C=F(\beta)\{\{\alpha\}\}[[\gamma]], D=F(\beta)\{\{\gamma\}\}[[\alpha]]$. Let $I$ be the ideal of $C$ generated by $\gamma$ and $J$ the ideal of $D$ generated by $\alpha$.

The Galois group of $f$ over $L$ is a subgroup (as a group of permutations on the roots) of its group over $F(\alpha, \beta, \gamma)$.

Factor $f(X)$ into $f_{1}(X) f_{2}(X)$ over $A$ as in Lemma 7 and let $\alpha_{1}, \ldots, \alpha_{k}$ be the roots of $f_{1}(X), \alpha_{k+1}, \ldots, \alpha_{n}$ those of $f_{2}(X)$. We regard $f_{2}(X)$ as a polynomial in $C[X]$ and reduce modulo $I$, getting a polynomial with distinct roots $\bar{f}_{2}(X)=\bar{\alpha} X^{n-k}+\bar{\beta}$ by the assumption on the characteristic. The group of this polynomial has an element which permutes the roots cyclicly, hence so does the group of $f_{2}(X)$ by the theorem of p. 190 of [3]. (Although $f_{2}(X)$ is not monic, its leading coefficient is a unit in $C$ so it may be treated as monic.)

Now we replace $X$ by $1 / Y$ in $f_{1}(X)$ and multiply by $Y^{k}$ to get $g_{1}(Y) \in$ $A[Y], g_{1}(Y) \equiv 1+(\gamma / \beta) Y^{k}(\bmod \alpha \gamma)$ whose roots are the reciprocals of those of $f_{1}(X)$. Regarding $g_{1}(Y)$ as an element of $D[Y]$ and reducing modulo $J$ we get a polynomial with distinct roots whose Galois group has an element which permutes them cyclicly. Hence the same is true for $g_{1}(Y)$, hence for $f_{1}(X)$. This completes the proof.

Remark. By dividing by $\alpha$ to get $X^{n}+(\beta / \alpha) X^{k}+(\gamma / \alpha)$ we see that we may apply Lemma 8 to the equation, $X^{n}+a X^{k}+b$, where $a$ and $b$ are independent transcendentals.

We now state precisely and prove the main result.

THEOREM. Let the characteristic of the field $F$ be prime to $k, n$ and $n-k$, and suppose $(n, k)=1$. Let $a, b$ be independent over $F$. Then the Galois group of $f(X)=X^{n}+a X^{k}+b$ over $K=F(a, b)$ is the full symmetric group on the roots.

Proof. The group is transitive by Lemma 2 and hence primitive by Lemmas 6 and 8. Since it cortains a transposition by Lemma 5 it is $S_{n}$ by Theorem 13.3 of [4].

The above leads to the conjecture that, subject to appropriate restrictions on the characteristic of $F$, the Galois group of $F(X)=X^{n}+\sum_{I} a_{i} X^{i}$ over $K=F\left(a_{i}, i \in I\right)$ is the full symmetric group, where $I$ is any subset of $\{0, \ldots, n-1\}$ containing 0 and such that g.c.d. $(n, i, i \in I)=1$, and the $a_{i}$ are independent over $F$. By taking specializations one can use the above theorem to show that this is the case if some $i \in I$ is prime to $n$; the smallest uncovered case is thus $f(X)=X^{6}+a X^{3}+b X^{2}+c$.

Note ADDED IN PROOF. See also P. Lefton, On the Galois groups of cubics and trinomials, Bull. Amer. Math. Soc. 82 (1976), 754-755, and the references cited there. 


\section{REFERENCES}

1. N. Bourbaki, Elements of mathematics. Commutative algebra, Hermann, Paris; AddisonWesley, Reading, Mass., 1972. MR 50 \# 12997.

2. K. Uchida, Galois group of an equation $X^{n}-a X+b=0$, Tôhoku Math. J. (2) 22 (1970), 670-678. MR 43 \#3238.

3. B. L. van der Waerden, Modern algebra, Vol. 1, Springer-Verlag, Berlin, 1937; rev. English transl., Ungar, New York, 1953. MR 2, 120; 10, 587.

4. H. Wielandt, Finite permutation groups, Lectures, Univ. of Tübingen, 1954/55; English transl., Academic Press,New York, 1964. MR 32 \# 1252.

Department of Mathematics, Boston College, Chestnut Hill, Massachusetts 02167 